\title{
Modelling of Heating and Evaporation of Gasoline Fuel Droplets: A Comparative Analysis of Approximations
}

\author{
A.E. Elwardany ${ }^{1 *}$, S.S. Sazhin ${ }^{2}$, A. Farooq ${ }^{1}$ \\ ${ }^{1}$ Clean Combustion Research Centre, Division of Physical Sciences and Engineering, King Abdullah \\ University of Science and Technology (KAUST), Thuwal 23955-6900, Saudi Arabia \\ ${ }^{2}$ Sir Harry Ricardo Laboratories, Centre for Automotive Engineering, School of \\ Computing, Engineering and Mathematics, Faculty of Science and Engineering, \\ University of Brighton, Brighton, BN2 4GJ, UK
}

FUEL (in press)

\begin{abstract}
Modelling of gasoline fuel droplet heating and evaporation processes is investigated using several approximations of this fuel. These are quasi-components used in the quasi-discrete model and the approximations of these quasi-components (Surrogate I (molar fractions: $83.0 \% n$ $\mathrm{C}_{6} \mathrm{H}_{14}+15.6 \% n-\mathrm{C}_{10} \mathrm{H}_{22}+1.4 \% n-\mathrm{C}_{14} \mathrm{H}_{30}$ ) and Surrogate II (molar fractions: $83.0 \% n-\mathrm{C}_{7} \mathrm{H}_{16}+$ $15.6 \% n-\mathrm{C}_{11} \mathrm{H}_{24}+1.4 \% n-\mathrm{C}_{15} \mathrm{H}_{32}$ )). Also, we have used Surrogate A (molar fractions: $56 \% n$ $\mathrm{C}_{7} \mathrm{H}_{16}+28 \%$ iso- $\mathrm{C}_{8} \mathrm{H}_{18}+17 \% \mathrm{C}_{7} \mathrm{H}_{8}$ ) and Surrogate B (molar fractions: $63 \% n-\mathrm{C}_{7} \mathrm{H}_{16}+20 \%$ iso$\mathrm{C}_{8} \mathrm{H}_{18}+17 \% \mathrm{C}_{7} \mathrm{H}_{8}$ ), originally introduced based on the closeness of the ignition delay of surrogates to that of gasoline fuel. The predictions of droplet radii and temperatures based on three quasi-components and their approximations (Surrogates I and II) are shown to be much more accurate than the predictions using Surrogates A and B.
\end{abstract}

\section{Introduction}

Gasoline fuel is widely used in automotive and industrial applications [1]. In Gasoline Direct Injection 'GDI' engines, the fuel is supplied to the combustion chamber during the compression stroke in the form of sprays [2], which consist of droplet streams. Droplets in these streams are broken up, heated and evaporated, and these processes

Keywords:

Gasoline fuel, multi-component droplets, gasoline surrogates, heating, evaporation, modelling

${ }^{*}$ Corresponding author. Tel. +966 (0) 2 808-4658; E-mail Ahmed.Elwardani@kaust.edu.sa 
take place almost simultaneously. They affect the mixture preparation (air + fuel vapour) and the physical ignition delay [3].

Models for heating and evaporation of fuel droplets can be classified based on fuel composition: models based on the assumption that fuel is mono-component and those taking into account the multi-component composition of the fuel. Models taking into account the multicomponent composition of fuel are very important and relevant for practical applications and our analysis will be focused upon them. This group of models can be further subdivided into two subgroups: those applicable for a small number of components (Discrete Component Model (DCM)) [4]-[15] and those applicable for large numbers of components (e.g. Continuous Thermodynamics approach [16]-[23] and the Distillation Curve Model [24]). In the first subgroup, the choice of discrete components could be based on either the pseudo-components or the key components approach [16]. The pseudo-components approach is based on replacing the continuous distribution of the mixture with a discrete one. The key components approach is based on replacing the continuous mixture with components with thermodynamics properties similar to those of the continuous mixture [16]. In the second subgroup a number of additional simplifying assumptions are used including the assumption that species mix infinitely quickly inside the droplet or do not mix at all.

In $[3,25]$, it was pointed out that the effects of finite thermal conductivity and recirculation inside the droplets need to be taken into account in modelling droplet heating and evaporation. The simplest approach to doing this can be based on the application of the Effective Thermal Conductivity (ETC) model [28,29]. Also, in [12,13], which focused on the modelling of heating and evaporation of bi-component droplets, it was demonstrated that the effect of finite diffusivity and recirculation of liquid species cannot be ignored. These effects were taken into account based on the Effective Diffusivity (ED) model.

A quasi-discrete model for heating and evaporation of complex mixtures with large numbers of components was suggested in [26]. This model is based on replacing a large number of actual components with a small number of quasi-components to approximate complex mixtures. The version of this model developed in [26] is based on the assumption that fuel consists only of alkanes $\left(\mathrm{C}_{n} \mathrm{H}_{2 n+2}\right)$. These quasi-components are then treated as actual components with averaged values of the carbon number $n$, taking into account the effect of finite 
thermal conductivity and species diffusivities within the liquid droplet. It was assumed that the liquid density, viscosity, heat capacity and thermal conductivity of all quasi-components are the same as those for $n$-dodecane, while the values of latent heat of evaporation and saturated vapour pressure take into account the effect of the number of carbon atoms as suggested in [21]. This model was applied for the analysis of heating and evaporation of Diesel fuel approximated by 21 components. These correspond to a maximum of 20 quasi-components, having average properties between those for neighbouring alkanes with the difference between carbon numbers equal to 1 . It was demonstrated that the predictions of the model with 5 quasi-components are very close to the predictions of the model that includes contributions from all 20 quasicomponents.

In [27], the original quasi-discrete model was generalised to take into account the dependence of liquid density, viscosity, heat capacity and thermal conductivity on the number of carbon atoms. The new model was applied to the analysis of heating and evaporation of both Diesel (approximated by 21 components or maximum 20 quasi-components) and gasoline (approximated by 14 components or maximum 13 quasi-components) fuels. As in [26], it was demonstrated that in the case of Diesel fuel the results predicted using 5 quasi-components are almost indistinguishable from the results predicted by the model taking into account the contribution of all 20 quasi-components. In the case of gasoline fuel the results predicted using 3 quasi-components were very close to the results predicted by the model taking into account the contribution of 13 quasi-components. This demonstrates the potential usefulness of the quasidiscrete model for the analysis of heating and evaporation of droplets consisting of many components.

A number of authors have attempted to approximate gasoline fuels with various surrogates based on fuel ignition characteristics. In [30] the autoignition characteristics of gasoline/air and ternary surrogate/air mixtures behind reflected shock waves in conditions similar to those found in homogeneous charge compression ignition (HCCI) engine cycles were studied. Two surrogates were considered: Surrogate A (molar fractions: $56 \% \mathrm{C}_{7} \mathrm{H}_{16}+28 \% \mathrm{C}_{8} \mathrm{H}_{18}$ $+17 \% \mathrm{C}_{7} \mathrm{H}_{8}$ ) and Surrogate B (molar fractions: $63 \% \mathrm{C}_{7} \mathrm{H}_{16}+20 \% \mathrm{C}_{8} \mathrm{H}_{18}+17 \% \mathrm{C}_{7} \mathrm{H}_{8}$ ). The range of experiments covered combustion of fuel in air for lean, stoichiometric, and rich mixtures $(\Phi=0.5,1.0,2.0)$, two pressure ranges (15-25 and 45-60 atm), temperatures from 850 
to $1280 \mathrm{~K}$, and exhaust gas recirculation (EGR) loadings of 0, 20, and 30\%. Strong agreement was seen between ignition delay time measurements for RD387 gasoline and Surrogate B fuels. In our paper, the feasibility of using Surrogates A or B for the analysis of gasoline fuel droplet heating and evaporation will be investigated. We anticipate that our conclusions could be potentially extended to other surrogate approximations of gasoline fuels, based on the ignition characteristics (e.g. [31]).

The main focus of this paper is to perform an analysis of heating and evaporation of gasoline fuel droplets, assuming that this fuel can be approximated by the above-mentioned surrogate fuels, inferred from the study of fuel ignition characteristics, and by the set of quasicomponents, inferred from the quasi-discrete model. The models used in our analysis are briefly described in Section 2. The results of the application of these models to the analysis of heating and evaporation of gasoline fuel droplets are presented and discussed in Section 3. The most important results are summarized in Section 4.

\section{Model}

\subsection{Liquid Heat and Mass Transfer}

Our analysis is based on the analytical solutions to the one-dimensional heat conduction and species diffusion equations inside spherically-symmetric droplets implemented in the numerical code $[25,28,12]$.

The Effective Thermal Conductivity/Effective Diffusivity (ETC/ED) model has been used in our analysis [12,29]. This model takes into account the effect of finite thermal and mass diffusivities alongside the effect of recirculation inside the droplet [12].

The effects of coupling between the droplet and the surrounding gas and the effects of thermal radiation are ignored at this stage.

\subsection{Quasi-discrete model}

Following [26,27], the molar mass is used to describe the continuous distribution of gasoline fuel which is assumed to consist only of $n$-alkanes $\mathrm{C}_{n} \mathrm{H}_{2 n+2}$. The molar mass $(\mathrm{kg} / \mathrm{kmole})$ of $n$-alkane components is related to the number of carbon atoms $n$ as: 


$$
M(n)=14 n+2
$$

The distribution function was taken as [21]:

$$
f_{m}(n)=C_{m}\left(n_{0}, n_{f}\right) \frac{(M(n)-\gamma)^{\alpha-1}}{\beta^{\alpha} \Gamma(\alpha)} \exp \left[-\left(\frac{M(n)-\gamma}{\beta}\right)\right],
$$

where $n_{0}=5 \leq n \leq n_{f}=18$, subscripts 0 and $f$ stand for initial and final values of $n, \Gamma(\alpha)$ is the Gamma function, $\alpha=5.7$ and $\beta=15$ are parameters that determine the shape of the distribution (they are taken to be the same as in [21]), $\gamma$ is the origin of the distribution function and is set to 0 , and $C_{m}$ is the normalisation constant and is calculated based on Eq. (4) of [27]. $f_{m}$ is defined for the range of $n$ between $n_{0}$ and $n_{f}$ and is 0 outside this range. The distribution function was chosen to reproduce the distillation curve for gasoline as specified in ASTM D439 [17]. Following [21], it was truncated at $n$-pentane and it was assumed that gasoline fuel consists only of $n$-alkanes with $18 \geq n \geq 5$ for liquid fuels. This distribution function is shown in Fig. 1 .

The dependence of the latent heat of evaporation, vapour pressure, liquid density, viscosity, specific heat capacity and thermal conductivity on the number of carbon atoms are taken into account as in [27].

Following [26,27], the continuous distribution (2) is replaced with a discrete one, consisting of $N_{f}$ quasi-components with carbon numbers:

$$
\bar{n}_{j}=\frac{\int_{n_{j-1}}^{n_{j}} n f_{m}(n) \mathrm{d} n}{\int_{n_{j-1}}^{n_{j}} f_{m}(n) \mathrm{d} n} .
$$

The corresponding molar and mass fractions are found as

$$
\begin{aligned}
& X_{j}=\int_{n_{j-1}}^{n_{j}} f_{m}(n) \mathrm{d} n, \\
& Y_{j}=\frac{M\left(\bar{n}_{j}\right) X_{j}}{\sum_{j=1}^{j=N_{f}}\left[M\left(\bar{n}_{j}\right) X_{j}\right]},
\end{aligned}
$$

where $j$ is an integer in the range $1 \leq j \leq N_{f}$.

Equations (3-5) are applied to each quasi-component using relevant values of $n_{j}$ and $n_{j-1}$. 
The diffusion coefficient between quasi-components is assumed to be the same for all quasi-components and it is calculated using Eq. (22) of [26]. The viscosity of liquid gasoline fuel, used in the ETC/ED model, is assumed to be the same as for $n$-octane [36].

$$
\mu_{g f}=0.001 * 10^{\left(-5.9245+8.8809 \times 10^{2} \times T+1.2955 \times 10^{-2} \times T^{-1}-1.3596 \times 10^{-5} \times T^{2}\right)} .
$$

All other thermo-physical properties of the mixture and the vapour diffusion coefficient are calculated as in [12]. The properties of the quasi-components in the quasi-discrete model are the same as discussed in [27]. The thermo-physical properties of n-heptane and iso-octane, two components used in Surrogates A and B, are assumed to be the same as in [33] and [34], respectively. The physical properties of toluene, the third component used in Surrogate A and B, are taken from $[35,36]$. The vapour pressure for each component was calculated based on the Clausius-Clapeyron equation [12].

\section{Results}

A plot of $f_{m}(n)$ versus $n$ for gasoline fuel, using values of parameters $(\alpha, \beta, \gamma)$ given in $[21,27]$, is shown in Fig. 1. As can be seen from this figure, the value of $n$ for which $f_{m}(n)$ is maximal is equal to 5 . The average value of $n$ is estimated as $\bar{n}_{j}=\mathbf{7 . 0 5}$, which is reasonably close to $n=8$ (n-octane), a commonly used approximation for gasoline fuel.

We focus on a comparative analysis of the effects of various approximations of gasoline fuel, discussed in Section 2, on fuel droplet heating and evaporation. The following approximations were considered. Firstly we looked at the approximation of gasoline fuel by three quasi-components (molar fractions: 83.0\% $n-\mathrm{C}_{6.26} \mathrm{H}_{14.58}+15.6 \% n-\mathrm{C}_{10.24} \mathrm{H}_{22.48}+1.4 \% n$ $\mathrm{C}_{14.42} \mathrm{H}_{30.84}$ ) as suggested in [27] (see Fig. 1 and Table 1). Secondly, we considered the approximations of these quasi-components by two surrogates with $n$ rounded up and down to the nearest integers: Surrogate I (mole fractions: 83.0\%n- $\mathrm{C}_{6} \mathrm{H}_{14}+15.6 \% n-\mathrm{C}_{10} \mathrm{H}_{22}+1.4 \% n$ $\mathrm{C}_{14} \mathrm{H}_{30}$ ), Surrogate II (molar fractions: $83.0 \% n-\mathrm{C}_{7} \mathrm{H}_{16}+15.6 \% n-\mathrm{C}_{11} \mathrm{H}_{24}+1.4 \% n-\mathrm{C}_{15} \mathrm{H}_{32}$ ) (see Table 1). These surrogates allowed us to investigate the sensitivity of the results to the choice of the values of the number of carbon atoms for each quasi-component $\left(\bar{n}_{1}, \bar{n}_{2}\right.$ and $\left.\bar{n}_{3}\right)$. Also, this rounding up or down of the values of the carbon numbers would allow us to use these approximations of quasi-components in Computational Fluid Dynamics (CFD) codes, which do 
not recognise substances with non-integer $n$. Thirdly, we looked at the approximations of gasoline fuel by gasoline Surrogates A and B considered in [30] (see Table 1). Surrogates of the third group were chosen based of their ignition characteristics. Nobody, to the best of our knowledge, has explored the usefulness of these surrogates in investigation of gasoline fuel droplet heating and evaporation. The results obtained based on the above approximations will be compared with the results based on the approximation of gasoline fuel by 13 quasi-components and the simplistic case of one quasi-component, both based on the distribution function shown in Fig. 1, as discussed in [27].

The plots of droplet surface temperatures $T_{S}$ and radii $R_{d}$ versus time for the five surrogates, shown in Table 1, and the approximations of gasoline fuel by $1\left(n-\mathrm{C}_{7.05} \mathrm{H}_{16.1}\right)$ and 13 quasi-components are presented in Fig. 2. The initial droplet radii and temperatures are assumed equal to $10 \mu \mathrm{m}$ and $300 \mathrm{~K}$ respectively in all cases; the droplet velocities are assumed to be constant and equal to $10 \mathrm{~m} / \mathrm{s}$, while gas temperature and pressure are assumed equal to $450 \mathrm{~K}$ and 0.3 MPa respectively. The calculations were performed using the ETC/ED model. It can be seen from this figure that the predicted temporal variations of droplet surface temperatures and radii for Surrogates A and B and approximation of gasoline fuel with 1 quasi-component are qualitatively different from the predictions taking into account the contributions of all 13 quasicomponents, assumed to be the most accurate approximation of this fuel. It can be seen that Surrogates A and B behave as almost one component and this can be attributed to the closeness of the boiling points of the individual components of these surrogates. At the same time, the predictions for gasoline fuel by 3 quasi-components and Surrogates I and II are reasonably close to the predictions based on consideration of 13 quasi-components. This closeness is particularly visible in the case of Surrogate II.

The choice of 3 quasi-components and Surrogates I and II was made to perform a 'likewith-like' comparison with the model based on Surrogates A and B, based on three components. We appreciate that the approximation of realistic gasoline fuel by three quasi-components used in this paper has a number of limitations. Firstly, this approximation, based on the quasi-discrete model, does not take into account the contribution of other components apart from $n$-alkanes. Secondly, the approximation of the actual molar fractions of the $n$-alkane components by distribution (2) could be rather crude. The first limitation is unavoidable due to the current state 
of development of the quasi-discrete model. The second limitation could be avoided if a more accurate approximation were available. Whilst these limitations exist we have no other choice but to use the distribution used in [21,27].

The longest evaporation time and highest droplet surface temperature at the end of evaporation is predicted for the 13 quasi-components mixture. This is due to the contribution of heavy alkanes in the mixture. It is further observed that there are 3 heating-up periods for the approximation based on 3 quasi-components and Surrogates I and II. These periods are not observed for other mixtures. This can be attributed to the fact that the approximation based on 3 quasi-components and Surrogates I and II contains hydrocarbons with a wide range of carbon atoms, in contrast to other mixtures. The wide range of carbon atoms leads to significant differences in the thermo-physical properties of the mixture components $[26,27]$.

The limitations of using 1 quasi-component for the analysis of gasoline fuel droplet heating and evaporation were discussed earlier in [27]. As follows from Fig. 2, the replacement of 1 quasi-component with Surrogates A or B does not improve the situation. Hence, although Surrogates A and B can be used for approximation of the autoignition characteristics of gasoline fuel, they are not suitable approximations for the analysis of gasoline fuel droplet heating and evaporation.

Also, one can see from Fig. 2 that the predicted surface temperatures and radii are rather sensitive to the values of the carbon numbers, which is illustrated by the difference in predictions of these parameters by the approximations of gasoline fuel by 3 quasi-components and Surrogates I and II. This could potentially open the way to improving the predictions of the quasi-discrete models by replacing the approximation based on 3 quasi-components with noninteger $n$ with Surrogate II. However, further investigations would be required before we are able to issue this recommendation. This would include the analysis of possible contributions of other hydrocarbons (apart from $n$-alkanes) in rigorous approximations of gasoline fuels, and the analysis of fuel droplet heating and evaporation for a wide range of parameters. Before this is done, our analysis will focus primarily on the approximation based of 3 quasi-components, as in [27]. 
The plots of liquid molar fractions at the surface of the droplets $X_{l s, j=1,2,3}$ versus time for all 3 quasi-components are shown in Fig. 3. The values of $X_{l s, 1}$ (lighter component) and $X_{l s, 3}$ (heavier component) monotonically decrease and increase with time respectively. The behaviour of $X_{l s, 2}$ is different where it initially increases with time and then starts to decrease with time after the lighter component has fully evaporated. This behaviour is similar to that shown in Fig 11 of [27] for 4 quasi-components. It is consistent with our observation that the model based on 3 quasi-components predicts longer evaporation times and larger droplet surface temperatures at the final stages of droplet evaporation, compared with the model using 1 quasi-component.

\section{Conclusions}

Effects of fuel approximations and initial values of various parameters on heating and evaporation of gasoline droplets are investigated. The following approximations are considered: 3 quasi-components introduced in the quasi-discrete model and their approximations: Surrogate I (molar fractions: $83 \% n-\mathrm{C}_{6} \mathrm{H}_{14}+15.6 \% n-\mathrm{C}_{10} \mathrm{H}_{22}+1.4 \% n-\mathrm{C}_{14} \mathrm{H}_{30}$ ) and Surrogate II (molar fractions: $\left.83 \% n-\mathrm{C}_{6} \mathrm{H}_{14}+15.6 \% n-\mathrm{C}_{10} \mathrm{H}_{22}+1.4 \% n-\mathrm{C}_{14} \mathrm{H}_{30}\right)$ ), surrogate approximations of gasoline fuel based on its ignition characteristics: Surrogate A (molar fractions: $56 \%{ }_{n}-\mathrm{C}_{7} \mathrm{H}_{16}+$ $28 \%$ iso $-\mathrm{C}_{8} \mathrm{H}_{18}+17 \% \mathrm{C}_{7} \mathrm{H}_{8}$ ) and Surrogate B (molar fractions: $63 \% n-\mathrm{C}_{7} \mathrm{H}_{16}+20 \%$ iso- $\mathrm{C}_{8} \mathrm{H}_{18}+$ $17 \% \mathrm{C}_{7} \mathrm{H}_{8}$ ). The results are compared with the predictions of the model based on the approximation of gasoline fuel by 13 and 1 quasi-components. It has been shown that the predictions of the quasi-discrete model based on the approximation of gasoline fuel with three quasi-components, especially Surrogate II, are much more accurate than those based on other surrogate approximations compared with the predictions of the quasi-discrete model based on 13 quasi-components. This demonstrates the limitation of using fuel surrogates, proposed based on fuel ignition characteristics, for the analysis of liquid fuel droplet heating and evaporation.

\section{Acknowledgements}

The authors acknowledge EPSRC in the UK (grants EP/H001603/1 and EP/J006793/1) and the Clean Combustion Center of King Abdullah University of Science and Technology for financial support.

\section{References}


[1] Heywood JB. Internal combustion engines fundamentals. New York: McGraw-Hill Book Company; 1988.

[2] Basshuysen RV. Gasoline engine with direct injection: processes, systems, development, potential, $1^{\text {st }}$ ed. GWV Fachverlage GmbH, Wiesbaden (978- 8348-0670-3); 2009.

[3] Sazhina EM, Sazhin SS, Heikal MR, Babushok VI, Johns R. A detailed modelling of the spray ignition process in Diesel engines. Combust Sci Technol 2000;160:317-44.

[4] Abraham J, Magi V. A model for multicomponent droplet vaporization in sprays, SAE 1998; Paper No. 980511.

[5] Aggarwal SK, Mongia HC. Multicomponent and high-pressure effects on droplet vaporization, ASME J Eng Gas Turb Power 2002;124:248-55.

[6] Brenn G, Deviprasath LJ, Durst F, Fink C. Evaporation of acoustically levitated multi component liquid droplets, Int J Heat Mass Transfer 2007;50:5073-86.

[7] Ghosh J, Mukhopadhyay A, Rao GV, Sanyal D. Analysis of evaporation of dense cluster of bicomponent fuel droplets in a spray using spherical cell model, Int $\mathrm{J}$ Therm Sci 2008;47:584-90.

[8] Maqua C, Castanet G, Lemoine F. Bi-component droplet evaporation: temperature measurements and modelling, Fuel 2008;87:2932-42.

[9] Sirignano WA, Wu G. Multicomponent-liquid-fuel vaporization with complex configuration, Int J Heat Mass Transfer 2008;51:4759-74.

[10] Strotos G, Gavaises M, Theodorakakos A, Bergeles G. Influence of species concentration on the evaporation of suspended multicomponent droplets. ILASS 2008; Lake Como, Italy.

[11] Ra Y, Reitz RD. A vaporization model for discrete multi-component fuel sprays. Int J MultiPhase Flow 2009;35:101-17.

[12] Sazhin SS, Elwardany A, Krutitskii PA, Castanet G, Lemoine F, Sazhina EM, et al. A simplified model for bi-component droplet heating and evaporation. Int $\mathrm{J}$ Heat Mass Transfer 2010;53:4495-505.

[13] Sazhin SS, Elwardany A, Krutitskii PA, Deprédurand V, Castanet G, Lemoine F. Multicomponent droplet heating and evaporation: numerical simulation versus experimental data. Int J Therm Sci 2011;50:1164-80.

[14] Strotos G, Gavaises M, Theodorakakos A, Bergeles G. Numerical investigation of the evaporation of two-component droplets, Fuel 2011;90:1492-507. 
[15] Elwardany AE, Gusev IG, Castanet G, Lemoine F, Sazhin SS. Mono- and multi-component droplet cooling/heating and evaporation: comparative analysis of numerical models, Atom Sprays 2011;21(11):907-31.

[16] Ratzsch MT. Continuous thermodynamics. Pure Appl Chem 1989;61-6:1105-14.

[17] Tamim J, Hallett WLH. Continuous thermodynamics model for multicomponent vaporization. Chem Eng Sci 1995;50:2933-42.

[18] Lippert AM, Reitz RD. Modelling of multicomponent fuels using continuous distributions with application to droplet evaporation and sprays. SAE 1997; Technical Paper 972882.

[19] Hallett WLH. A simple model for the vaporization of droplets with large numbers of components. Combust Flame 2000;121:334-44.

[20] Zhu G-S, Reitz RD. A model for high-pressure vaporization of droplets of complex liquid mixture using continuous thermodynamics. Int J Heat Mass Transfer 2002;45:495-507.

[21] Arias-Zugasti M, Rosner DE. Multicomponent fuel droplet vaporization and combustion using spectral theory for a continuous mixture. Combust Flame 2003;135:271-84.

[22] Abdel-Qader Z, Hallett WLH. The role of liquid mixing in evaporation of complex multicomponent mixtures: modelling using continuous thermodynamics. Chem Eng Sci 2005;60:1629-40.

[23] Rivard E, Brüggemann D. Numerical investigation of semi-continuous mixture droplet vaporization. Chem Eng Sci 2010;65:5137-45.

[24] Burger M, Schmehl R, Prommersberger K, Schäfer O, Koch R, Wittig S. Droplet evaporation modelling by the distillation curve model: accounting for kerosene fuel and elevated pressures. Int J Heat Mass Transfer 2003;46:4403-12.

[25] Sazhin SS. Advanced models of fuel droplet heating and evaporation. Prog Energy Combust Sci 2006;32:162-214.

[26] Sazhin SS, Elwardany AE, Sazhina EM, Heikal MR. A quasi-discrete model for heating and evaporation of complex multi-component hydrocarbon fuel droplets, Int. J. Heat Mass Transfer, 2011;54:4325-32.

[27] Elwardany AE, Sazhin SS. A quasi-discrete model for heating and evaporation: application to Diesel and gasoline fuels, Fuel 2012;97:685-94.

[28] Sazhin SS, Krutitskii PA, Abdelghaffar WA, Sazhina EM, Mikhalovsky SV, Meikle ST and Heikal MR. Transient heating of diesel fuel droplets, Int. J. Heat Mass Transfer 2004;47:3327-40. 
[29] Abramzon B, Sirignano WA. Droplet vaporization model for spray combustion calculation, Int. J. Heat Mass Transfer 1989;32:1605-18.

[30] Gauthier BM, Davidson DF, Hanson RK. Shock tube determination of ignition delay times in full-blend and surrogate fuel mixtures. Comb Flame 2004; 139:300-11.

[31] Machrafi H, Cavadias S, Gilbert P. An experimental and numerical analysis of the HCCI auto-ignition process of primary reference fuels, toluene reference fuels and diesel fuel in an engine, varying the engine parameters. Fuel Processing Technology 2008; 89:1007-16.

[32] Poling BE, Prausnitz JM, O'Connell J. The properties of gases and liquids. New York: McGraw-Hill; 2000.

[33] Sazhin SS, Kristyadi T, Abdelghaffar WA, Heikal MR. Models for fuel droplet heating and evaporation: comparative analysis. Fuel 2006;85:1613-30.

[34] Kristyadi T. Modelling of the heating and evaporation of fuel droplets, $\mathrm{PhD}$ thesis, University of Brighton; 2007.

[35] Yaws CL. (Editor). Thermophysical properties of chemicals and hydrocarbons. William Andrew Inc; 2008.

[36] Yaws CL. (Editor). Transport properties of chemicals and hydrocarbons: viscosity, thermal conductivity, and diffusivity of $\mathrm{C} 1$ to $\mathrm{C} 100$ organics and $\mathrm{Ac}$ to $\mathrm{Zr}$ inorganics. William Andrew Inc. Published by Elsevier Inc, (2009).

[37] Bird RB, Stewart EW and Lightfoot EN. Transport Phenomena. $2^{\text {nd }}$ ed. New York, Chichester: Wiley and Sons; 2002.

[38] Abramzon B. and Sazhin S. Convective vaporization of fuel droplets with thermal radiation absorption. Fuel 2006; 85(1):32-46.

\section{Figure captions}

Fig. 1 A plot of $f_{m}(n)$ versus $n$ for gasoline fuel, as predicted by Eq. (19), showing the 3 quasi-components.

Fig. 2 Plots of droplet surface temperatures $T_{s}$ and radii $R_{d}$ versus time, predicted for 1,3 and 13 quasi-components used in the quasi-discrete model, the approximations of the 3 quasicomponents with Surrogates I and II (see Table 1) and Surrogates A and B (see Table 1). The initial droplet radii and temperatures are taken to be equal to $10 \mu \mathrm{m}$ and $300 \mathrm{~K}$ respectively, the droplet velocities equal to $10 \mathrm{~m} / \mathrm{s}$, and the gas temperature and pressure, $450 \mathrm{~K}$ and $0.3 \mathrm{MPa}$ respectively. The calculations were performed using the ETC/ED model.

Fig. 3 Plots of molar fraction $X_{l s}$ at the surface of the droplet for 3 quasi-components versus time, predicted for the same input parameters as in Fig. 2. 


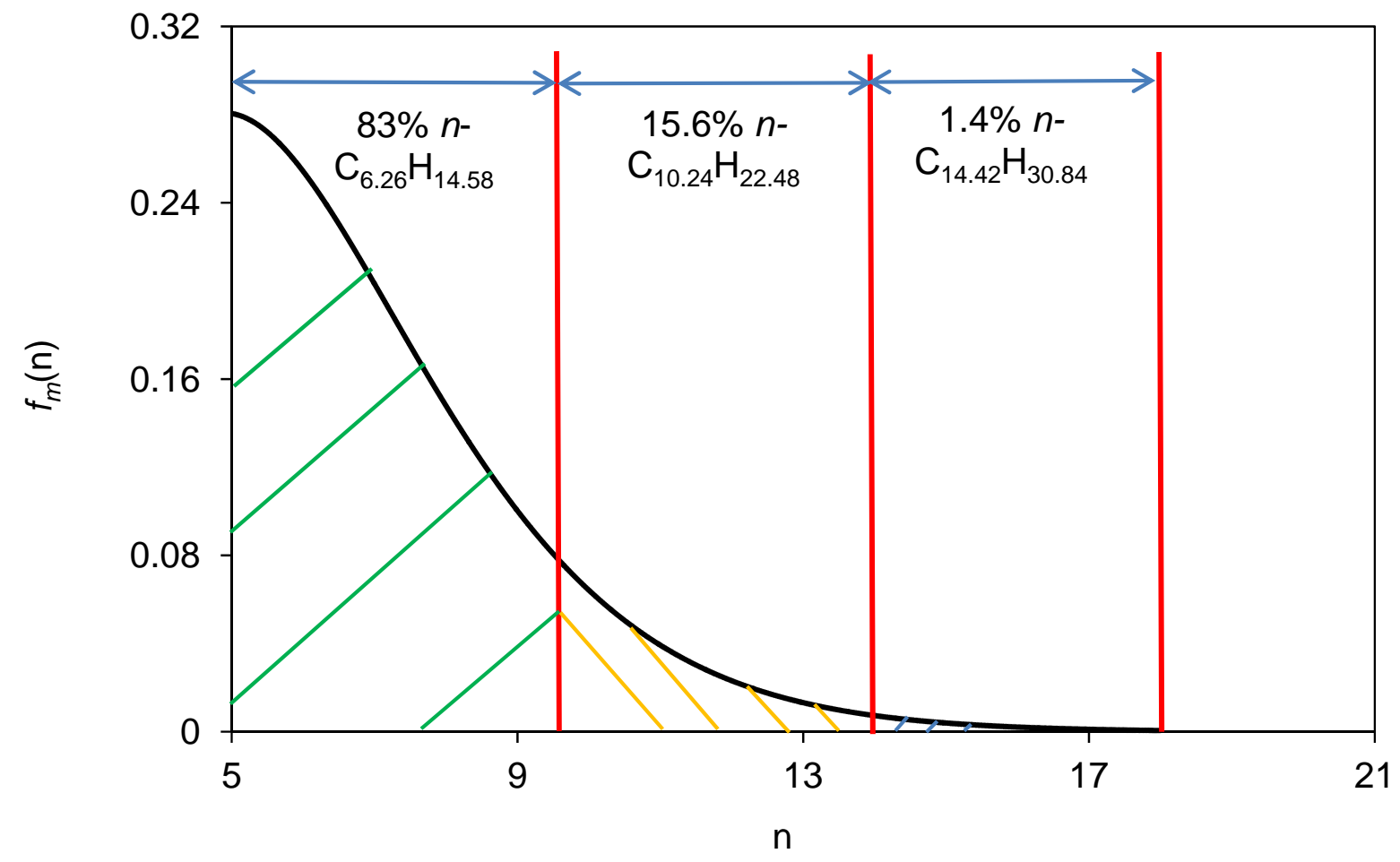

Fig. 1 


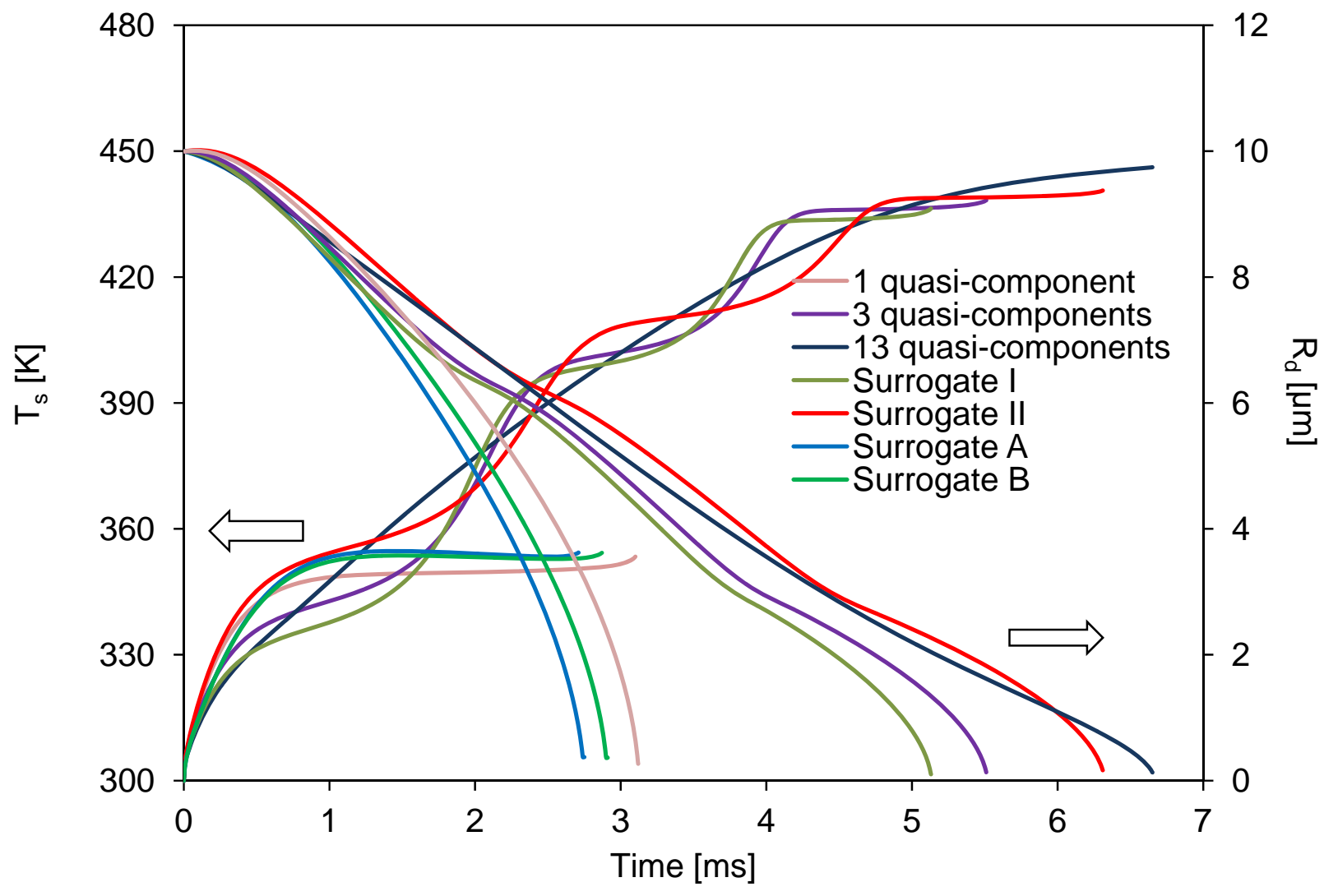

Fig. 2 


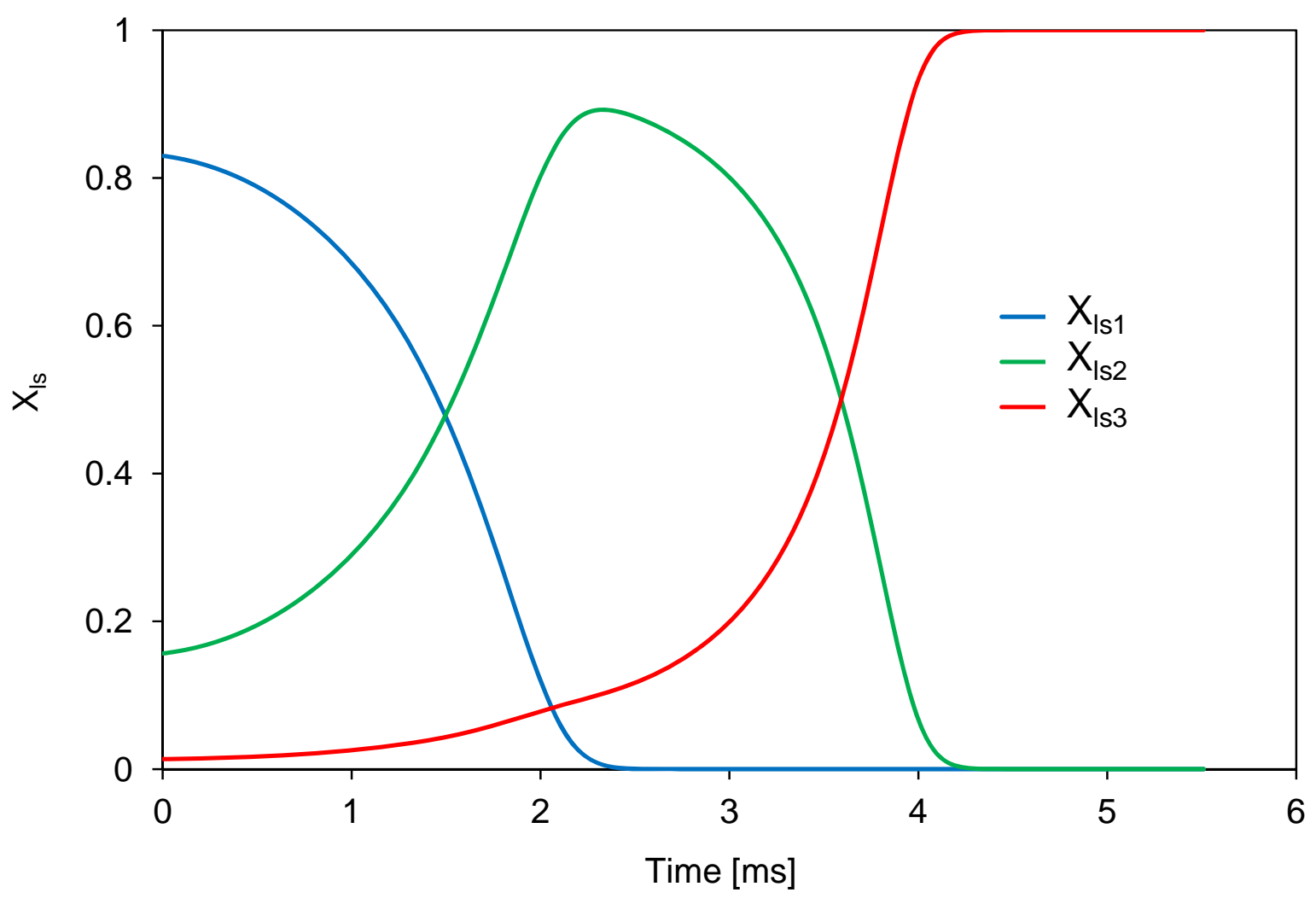

Fig. 3 


\section{Tables}

\begin{tabular}{lcl}
\hline Approximation & Ref. & Composition (molar fractions) \\
\hline 1 quasi-component & {$[27]$} & $n-\mathrm{C}_{7.05} \mathrm{H}_{16.1}$ \\
3 quasi-components & {$[27]$} & $83 \% n-\mathrm{C}_{6.26} \mathrm{H}_{14.58}+15.6 \% n-\mathrm{C}_{10.24} \mathrm{H}_{22.48}+1.4 \% n-\mathrm{C}_{14.42} \mathrm{H}_{30.84}$ \\
Surrogate I & & $83 \% n-\mathrm{C}_{6} \mathrm{H}_{14}+15.6 \% n-\mathrm{C}_{10} \mathrm{H}_{22}+1.4 \% n-\mathrm{C}_{14} \mathrm{H}_{30}$ \\
Surrogate II & new & $83 \% n-\mathrm{C}_{7} \mathrm{H}_{16}+15.6 \% n-\mathrm{C}_{11} \mathrm{H}_{24}+1.4 \% n-\mathrm{C}_{15} \mathrm{H}_{32}$ \\
Surrogate A & & $56 \% n-\mathrm{C}_{7} \mathrm{H}_{16}+28 \%$ iso- $\mathrm{C}_{8} \mathrm{H}_{18}+17 \% \mathrm{C}_{7} \mathrm{H}_{8}$ \\
Surrogate B & {$[30]$} & $63 \% n-\mathrm{C}_{7} \mathrm{H}_{16}+20 \%$ iso- $\mathrm{C}_{8} \mathrm{H}_{18}+17 \% \mathrm{C}_{7} \mathrm{H}_{8}$ \\
\hline
\end{tabular}

Table 1. Molar fractions of components in various gasoline fuel approximations and surrogates. 